\title{
A Multiuser Interference Cancellation Scheme for Uplink OFDMA
}

\author{
S. Manohar, V. Tikiya, D. Sreedhar, and A. Chockalingam \\ Department of ECE, Indian Institute of Science, Bangalore 560012, INDIA
}

\begin{abstract}
In uplink orthogonal frequency division multiple access (OFDMA) systems, multiuser interference (MUI) occurs due to different carrier frequency offsets (CFO) of different users at the receiver. In this paper, we present a multistage linear parallel interference cancellation (LPIC) approach to mitigate the effect of this MUI in uplink OFDMA. The proposed scheme first performs CFO compensation (in time domain), followed by $K$ DFT operations (where $K$ is the number of users) and multistage LPIC on these DFT outputs. We scale the MUI estimates by weights before cancellation and optimize these weights by maximizing the signal-to-interference ratio (SIR) at the output of the different stages of the LPIC. We derive closed-form expressions for these optimum weights. The proposed LPIC scheme is shown to effectively cancel the MUI caused by the other user CFOs in uplink OFDMA.
\end{abstract}

Keywords - Uplink OFDMA, carrier frequency offset, multiuser interference, interference cancellation, optimum weights.

\section{INTRODUCTION}

Recent research has been witnessing increased focus on orthogonal frequency multiple access (OFDMA) on the uplink [1]-[10]. The performance of OFDM/OFDMA systems depend to a large extent on how well the orthogonality among different subcarriers are maintained at the receiver [11],[12]. Factors including carrier frequency offsets (CFO) between the transmitter and receiver induced by Doppler effects and/or poor oscillator alignments, sampling clock frequency discrepancies, and time delay caused by multipath and non-ideal synchronization can destroy the orthogonality among subcarriers. Among the above factors, the impact of CFO on the performance is the most crucial one because the $\mathrm{CFO}$ values are large (typically of the order of several $\mathrm{KHz}$ ) due to carrier frequencies being of the order of GHz. In uplink OFDMA, correction to one user's CFO would misalign other initially aligned users. Thus, other user CFO will result in significant multiuser interference (MUI) in uplink OFDMA.

There have been a few recent attempts in the literature that address the issue of MUI due to other user CFO in uplink OFDMA [7]-[10]. The approach proposed in [7] is to feedback the estimated CFO values to the mobiles so that the mobile transmitters can adjust their transmit frequencies. This needs additional signaling and hence reduces system throughput. An alternate approach is to apply interference cancellation (IC) techniques at the base station receiver [8]-[10]. Recently, in [9], Huang and Letaief presented an IC approach which performs CFO compensation and MUI cancellation in frequency domain using circular convolution. We refer to this scheme in [9] as Huang-Letaief Circular Convolution (HLCC) scheme. The circular convolution approach was proposed earlier by Choi et al in [6] as an alternative to the di-

This work was supported in part by the Swarnajayanti Fellowship, Department of Science and Technology, New Delhi, Government of India, under Project Ref: No.6/3/2002-S.F. rect time-domain method of $\mathrm{CFO}$ compensation. Huang and Letaief refer the scheme in [6] as CLJL scheme (CLJL stands for the first letters of the names of the four authors of [6]). The CLJL scheme does not perform MUI cancellation. The HLCC scheme uses circular convolution for both CFO compensation (as in [6]) as well as MUI cancellation. In [10], we have proposed a minimum mean square error (MMSE) receiver for MUI cancellation in uplink OFDMA. We derived a recursion to approach the MMSE solution and showed that this recursive MMSE solution encompasses the CLJL and HLCC schemes as special cases.

Structure-wise, a common feature in CLJL [6], HLCC [9], and MMSE [10] schemes is that all these detectors/cancellers first perform a single DFT operation on the received samples and the resulting DFT output vector is further processed to achieve CFO compensation and MUI cancellation using circular convolution. A new contribution in this paper is that we propose and analyze an alternate MUI cancellation receiver structure which first performs CFO compensation in time domain, followed by $K$ DFT operations (where $K$ is the number of users) and multistage linear parallel interference cancellation (LPIC) on these DFT outputs. We scale the estimated MUI by weights before cancellation. For this proposed scheme, we drive closed-form expressions for the average signal-to-interference ratio (SIR) at the output of various stages of the LPIC. We also derive closed-form expressions for the optimum weights that maximize the average SIR at the output of the different LPIC stages. We show that the proposed weighted LPIC scheme effectively cancels the MUI caused by the other-user CFOs.

\section{UPLINK OFDMA SYSTEM MODEL}

We consider an uplink OFDMA system with $K$ users, where each user communicates with a base station through an independent multipath channel as shown in Fig. 1. We assume that there are $N$ subcarriers in each OFDM symbol and one subcarrier can be allocated to only one user. The information symbol for the $i$ th user on the $k$ th subcarrier is denoted by $X_{k}^{(i)}, k \in S_{i}$, where $S_{i}$ is the set of subcarriers assigned to user $i$ and $E\left[\left|X_{k}^{(i)}\right|^{2}\right]=1$. Then, $\bigcup_{i=1}^{K} S_{i}=$ $\{0,1, \ldots, N-1\}$ and $S_{i} \bigcap S_{j}=\phi$, for $i \neq j$. The length of the guard interval added is $N_{g}$ samples and is assumed to be longer than the maximum channel delay spread. After IDFT processing and guard interval insertion at the transmitter, the time domain sequence of the $i$ th user, $x_{n}^{(i)}$, is given by

$$
x_{n}^{(i)}=\frac{1}{N} \sum_{k \in S_{i}} X_{k}^{(i)} e^{\frac{j 2 \pi n k}{N}}, \quad-N_{g} \leq n \leq N-1 .
$$

The $i$ th user's signal, after passing through the channel, is 


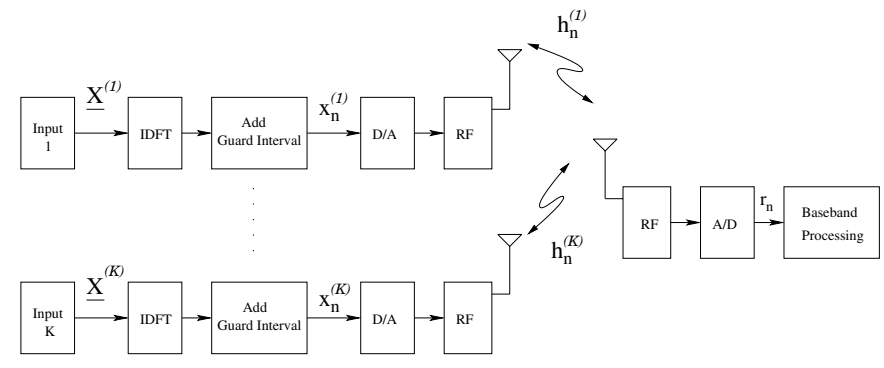

Fig. 1. Uplink OFDMA system model.

given by

$$
s_{n}^{(i)}=x_{n}^{(i)} \star h_{n}^{(i)}
$$

where $\star$ denotes linear convolution and $h_{n}^{(i)}$ is the $i$ th user's channel impulse response. It is assumed that $h_{n}^{(i)}$ is non-zero only for $n=0, \ldots, L-1$, where $L$ is the maximum channel delay spread, and that all users' channels are statistically independent. We assume that $h_{n}^{(i)}$ 's are i.i.d. complex Gaussian with zero mean and $E\left[\left(h_{n, I}^{(i)}\right)^{2}\right]=E\left[\left(h_{n, Q}^{(i)}\right)^{2}\right]=1 / L$, where $h_{n, I}^{(i)}$ and $h_{n, Q}^{(i)}$ are the real and imaginary parts of $h_{n}^{(i)}$. The channel coefficient in frequency domain $H_{k}^{(i)}$ is given by

$$
H_{k}^{(i)}=\sum_{n=0}^{L-1} h_{n}^{(i)} e^{\frac{-j 2 \pi n k}{N}},
$$

and $E\left[\left|H_{k}^{(i)}\right|^{2}\right]=2$. The received baseband signal after coarse carrier frequency tracking (leaving some residual carrier frequency offset) is given by

$$
r_{n}=\sum_{i=1}^{K} s_{n}^{(i)} e^{\frac{j 2 \pi \epsilon_{i} n}{N}}+z_{n}, \quad-N_{g} \leq n \leq N-1,
$$

where $\epsilon_{i}, i=1, \ldots, K$ denotes $i$ th user's residual carrier frequency offset (CFO) normalized by the subcarrier spacing, and $z_{n}$ is the AWGN with zero mean and variance $\sigma^{2}$. We assume that all users are time synchronized and that $\epsilon_{i}, i=$ $1, \cdots, K$ are known at the receiver.

Figure 2 shows the receiver baseband processing including $i$ ) CFO compensation in time domain and guard time removal, ii) $K$ DFT operations (one for each user), and iii) linear parallel interference cancellation (LPIC) in multiple stages. Note that the CFO compensation is carried out in time domain by multiplying $r_{n}$ with $e^{-\frac{j 2 \pi \epsilon_{i} n}{N}}, i=1, \cdots, K$ (this method of CFO compensation is referred to as the direct method in [6]). The received signal after CFO compensation and guard time removal for the $i$ th user is given by

$$
y_{n}^{(i)}=r_{n} e^{-\frac{j 2 \pi \epsilon_{i} n}{N}}, \quad 0 \leq n \leq N-1,
$$

which forms the input to the $i$ th DFT block. The output of the DFT block for the $i$ th user on the $k$ th subcarrier is then given by

$$
Y_{k}^{(i)}=H_{k}^{(i)} X_{k}^{(i)}+\sum_{\substack{l=1 \\ l \neq i}}^{K} \sum_{q \in S_{l}} \rho_{k q}^{(i),(l)} H_{q}^{(l)} X_{q}^{(l)}+Z_{k}^{(i)}
$$

where

$$
\rho_{k q}^{(i),(l)}=\frac{\sin \pi\left(k-q+\delta_{l i}\right)}{N \sin \frac{\pi}{N}\left(k-q+\delta_{l i}\right)} \exp \left(-j\left(1-\frac{1}{N}\right) \pi\left(k-q+\delta_{l i}\right)\right),
$$

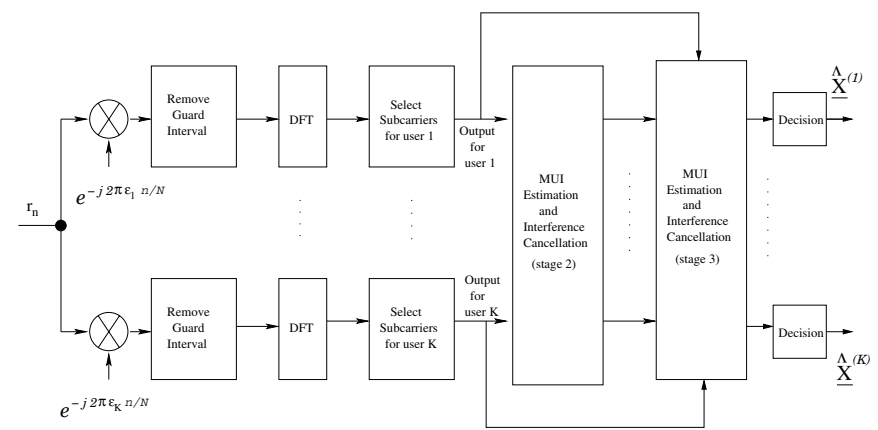

Fig. 2. Receiver baseband processing - $\mathrm{CFO}$ compensation (in time domain) and multistage interference cancellation.

and $\delta_{l i}$ is the difference between the $i$ th user and $l$ th user CFO values given by

$$
\delta_{l i}=\epsilon_{l}-\epsilon_{i} .
$$

The channel coefficient $H_{k}^{(i)}$ is given by (17) and the noise component $Z_{k}^{(i)}$ is given by

$$
Z_{k}^{(i)}=\sum_{n=0}^{N-1} z_{n}^{(i)} e^{\frac{-j 2 \pi n\left(k+\epsilon_{i}\right)}{N}}
$$

Note that the 2nd term in (6) represents the MUI present at the DFT output. In the case of single user detection (SUD), the DFT outputs, $Y_{k}^{(i)}$, s, can be directly used to make the symbol decision. Additional processing may be performed on $Y_{k}^{(i)}$, s to mitigate the effect of the MUI. For example, multistage interference cancellation techniques can be employed to improve performance. In the following section, we propose a multistage weighted linear parallel interference cancellation scheme which operates on the DFT outputs, $Y_{k}^{(i)}$ 's.

\section{Proposed Weighted Linear PIC Scheme}

The proposed multistage weighted linear PIC (WLPIC) scheme is explained as follows. Let $m$ denote the stage index. We take the DFT outputs, $Y_{k}^{(i)}$ s, in (6) as the first stage $(m=1)$ outputs of the receiver, i.e., $Y_{k,(1)}^{(i)}=Y_{k}^{(i)}$. In the case of SUD, the symbol decisions are made directly from $Y_{k,(1)}^{(i)}$ 's. Parallel interference cancellation is performed in the subsequent stages. In a given LPIC stage $m, m>1$, an estimate of the MUI is made based on the soft values of the previous stage outputs. These MUI estimates are scaled by weights and cancelled from the DFT outputs, $Y_{k,(1)}^{(i)}$.

The interference cancelled output of the $i$ th user on the $k$ th subcarrier in the $m$ th stage, $Y_{k,(m)}^{(i)}, m>1$, can be written as

$$
Y_{k,(m)}^{(i)}=Y_{k,(1)}^{(i)}-w_{k,(m)}^{(i)} \underbrace{\sum_{\substack{l=1 \\ l \neq i}}^{K} \sum_{q \in S_{l}} \rho_{k q}^{(i),(l)} Y_{q,(m-1)}^{(l)}}_{\text {MUI estimate }}
$$

where $Y_{k,(1)}^{(i)}$ is the 1st stage output given by (6) and $\rho_{k q}^{(i),(l)}$ is given by (7). It is noted that $\sum_{\substack{l=1 \\ l \neq i}}^{K} \sum_{q \in S_{l}} \rho_{k q}^{(i),(l)} Y_{q,(m-1)}^{(l)}$ is the MUI estimate, and $w_{k,(m)}^{(i)}$ is the weight with which this MUI estimate is scaled and cancelled. It is noted that the SUD becomes a special case of the proposed WLPIC scheme 
for $w_{k,(m)}^{(i)}=0, \forall i, k, m$. We call the WLPIC scheme with unity weights on all subcarriers (i.e., $\left.w_{k,(m)}^{(i)}=1, \forall i, k, m\right)$ as conventional LPIC (CLPIC) scheme. In the CLPIC scheme, the operations needed for choice of optimum weights and MUI scaling with these weights are avoided (because of unity weights). However, performance better than that of the CLPIC can be achieved by using optimum weights. We propose to obtain the optimum weights $w_{k,(m)}^{(i), o p t}$ by maximizing the average SIR at the $m$ th stage output.

In an uncoded system, the symbol decision for the $i$ th user on the $k$ th subcarrier at the output of the $m$ th stage can be made based on the output $Y_{k,(m)}^{(i)}$. For example, the symbol decision at the $m$ th stage output for the case of BPSK modulation can be obtained as

$$
\widehat{X}_{k,(m)}^{(i)}=\operatorname{sgn}\left(\mathcal{R} e\left(\overline{H_{k}^{(i)}} Y_{k,(m)}^{(i)}\right)\right),
$$

where $\overline{H_{k}^{(i)}}$ denotes the conjugate ${ }^{1}$ of $H_{k}^{(i)}$. For the case of $M$-QAM/ $M$-PSK modulation, symbol decision can be made using the minimum Euclidean distance rule. In a coded system, the $Y_{k,(m)}^{(i)}$ 's are fed to the decoder.

\section{SIR ANALYSIS}

Here, we derive expressions for the average SIR at the output of the 2nd and 3rd stages of the proposed WLPIC scheme. Also, we will use the derived average SIR expressions to obtain closed-form expressions for the optimum weights $w_{k,(m)}^{(i), o p t}$. It is noted that the average output SIR on a given subcarrier will depend on several things including number of users, channel impulse response, number of subcarriers, CFO values, and type of subcarrier allocation. Here, we consider two types of subcarrier allocation, namely, $a$ ) interleaved allocation and $b$ ) block allocation. In block allocation, a consecutive block of subcarriers are alloted to one user, the next block to another user, and so on. In interleaved allocation, the subcarriers of each user are uniformly interleaved with the subcarriers assigned to the other users.

\section{A. Average SIR at the 2nd Stage Output}

From (10), the weighted interference cancelled output of the 2 nd stage $(m=2)$ for the $i$ th user on the $k$ th subcarrier can be written as

$Y_{k,(2)}^{(i)}=H_{k}^{(i)} X_{k}^{(i)}\left(1-w_{k,(2)}^{(i)} \sum_{\substack{l=1 \\ l \neq i}}^{K} \sum_{q \in S_{l}} \rho_{k q}^{(i),(l)} \rho_{q k}^{(l),(i)}\right)+I_{2}+N_{2}$,

where

$$
\begin{gathered}
I_{2}=\sum_{l=1}^{K} \sum_{\substack{p \in S_{l} \\
p \neq k}} H_{p}^{(l)} X_{p}^{(l)}\left[\rho_{k p}^{(i),(l)}\left(1-w_{k,(2)}^{(i)}\right)\right. \\
\left.-w_{k,(2)}^{(i)} \sum_{\substack{u=1 \\
u \neq i, l}}^{K} \sum_{v \in S_{u}} \rho_{k v}^{(i),(u)} \rho_{v p}^{(u),(l)}\right], \\
N_{2}=Z_{k}^{(i)}-w_{k,(2)}^{(i)} \sum_{\substack{l=1 \\
l \neq i}}^{K} \sum_{q \in S_{l}} \rho_{k q}^{(i),(l)} Z_{q}^{(l)} .
\end{gathered}
$$

The terms $I_{2}$ and $N_{2}$ in (13) and (14) represent the interference and noise terms introduced due to imperfect cancellation

\footnotetext{
${ }^{1}$ We will use 'overline' to denote conjugate operation.
}

in using the soft output values from the first stage. After coherent combining using $\overline{H_{k}^{(i)}}$, the final output is given by

$$
\begin{aligned}
\overline{H_{k}^{(i)}} Y_{k,(2)}^{(i)}= & \left|H_{k}^{(i)}\right|^{2} X_{k}^{(i)}\left(1-w_{k,(2)}^{(i)} \sum_{\substack{l=1 \\
l \neq i}}^{K} \sum_{q \in S_{l}} \rho_{k q}^{(i),(l)} \rho_{q k}^{(l),(i)}\right) \\
& +I_{2}^{\prime}+N_{2}^{\prime},
\end{aligned}
$$

where $I_{2}^{\prime}=\overline{H_{k}^{(i)}} I_{2}$ and $N_{2}^{\prime}=\overline{H_{k}^{(i)}} N_{2}$.

Considering the $\overline{H_{k}^{(i)}} H_{p}^{(l)}$ factor in $I_{2}^{\prime}$, it is noted that the channel coefficients on subcarriers of different users $i 1$ and $i 2, H_{k 1}^{(i 1)}$ and $H_{k 2}^{(i 2)}, k 1 \in S_{i 1}, k 2 \in S_{i 2}, i 1 \neq i 2$, are uncorrelated because all users' channels are assumed to be independent. However, from (3), it can be seen that the channel coefficients on different subcarriers of the same user $i, H_{k 1}^{(i)}$ and $H_{k 2}^{(i)}, k 1, k 2 \in S_{i}$, are correlated. Also, this correlation depends on the subcarrier allocation. Handling the correlation between $H_{k 1}^{(i)}$ and $H_{k 2}^{(i)}$ in the SIR analysis is tedious. Therefore, to simplify the analysis, we assume that $H_{k 1}^{(i)}$ and $H_{k 2}^{(i)}$ are uncorrelated. Accordingly, the variance of $I_{2}^{\prime}, \sigma_{I_{2}^{\prime}}^{2}$, can be obtained as

$$
\sigma_{I_{2}^{\prime}}^{2}=\left|H_{k}^{(i)}\right|^{2} \sigma_{I_{2}}^{2}
$$

where

$$
\begin{aligned}
\sigma_{I_{2}}^{2}= & 2 \sum_{l=1}^{K} \sum_{\substack{p \in S_{l} \\
p \neq k}} \mid \rho_{k p}^{(i),(l)}\left(1-w_{k,(2)}^{(i)}\right) \\
& -\left.w_{k,(2)}^{(i)} \sum_{\substack{u=1 \\
u \neq i, l}}^{K} \sum_{v \in S_{u}} \rho_{k v}^{(i),(u)} \rho_{v p}^{(u),(l)}\right|^{2},
\end{aligned}
$$

and the variance of $N_{2}^{\prime}, \sigma_{N_{2}^{\prime}}^{2}$, can be obtained as

$$
\sigma_{N_{2}^{\prime}}^{2}=\left|H_{k}^{(i)}\right|^{2} \sigma_{N_{2}}^{2}
$$

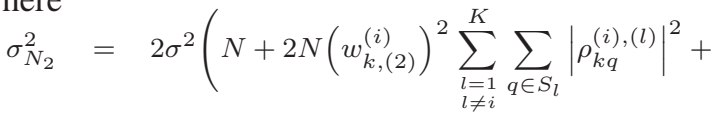

$$
\begin{aligned}
& \left(w_{k,(2)}^{(i)}\right)^{2} \sum_{\substack{l=1 \\
l \neq i}}^{K} \sum_{q \in S_{l}} \sum_{\substack{u=1 \\
u \neq i, l}}^{K} \sum_{v \in S_{u}} \rho_{k q}^{(i),(l)} \overline{\rho_{k v}^{(i),(u)}} \eta_{q v}^{(l),(u)} \\
& \left.-\mathcal{R} e\left[2 w_{k,(2)}^{(i)} \sum_{\substack{l=1 \\
l \neq i}}^{K} \sum_{q \in S_{l}} \rho_{k q}^{(i),(l)} \eta_{q k}^{(l),(i)}\right]\right),
\end{aligned}
$$

and

$$
\eta_{q k}^{(l),(i)}=E\left[Z_{q}^{(l)} \overline{Z_{k}^{(i)}}\right]=\sum_{n=0}^{N-1} e^{\frac{-j 2 \pi n\left(\epsilon_{l}-\epsilon_{i}+k-q\right)}{N}} .
$$

The average SIR on the $k$ th subcarrier of the $i$ th user at the output of the 2nd stage, $S I R_{k,(2)}^{(i)}$, can then be obtained as

$$
\operatorname{SIR}_{k,(2)}^{(i)}=\frac{2\left(1-w_{k,(2)}^{(i)} \sum_{\substack{l=1 \\ l \neq i}}^{K} \sum_{q \in S_{l}} \rho_{k q}^{(i),(l)} \rho_{q k}^{(l),(i)}\right)^{2}}{\sigma_{I_{2}}^{2}+\sigma_{N_{2}}^{2}}
$$

\section{B. Average SIR at the 3rd Stage Output}

The soft values of the interference cancelled outputs of the different users from the 2nd stage, $Y_{q}^{(l)}, l \neq i, q \in S_{l}$, are used to reconstruct (estimate) the MUI on the $k$ th subcarrier of the desired user $i$ in the 3 rd stage. The MUI estimates are 
weighted by $w_{k,(3)}^{(i)}$ and cancelled. Accordingly, the weighted interference cancelled output of the 3 rd stage, $Y_{k,(3)}^{(i)}$, is given by

$$
\begin{gathered}
Y_{k,(3)}^{(i)}=H_{k}^{(i)} X_{k}^{(i)} F+I_{3}+N_{3} \\
F=\quad 1-w_{k,(3)}^{(i)} \sum_{\substack{l=1 \\
l \neq i}}^{K} \sum_{q \in S_{l}} \rho_{k q}^{(i),(l)}\left(\rho_{q k}^{(l),(i)}\left(1-w_{q,(2)}^{(l)}\right)\right. \\
\left.-w_{q,(2)}^{(l)} \sum_{\substack{u=1 \\
u \neq 1, i}}^{K} \sum_{v \in S_{u}} \rho_{q v}^{(l),(u)} \rho_{v k}^{(u),(i)}\right) \\
N_{3}=Z_{k}^{(i)}-w_{k,(3)}^{(i)} \sum_{\substack{l=1 \\
l \neq i}}^{K} \sum_{q \in S_{l}} \rho_{k q}^{(i),(l)}\left(Z_{q}^{(l)}\right. \\
\left.I_{3}=\sum_{l=1}^{K} \sum_{\substack{q \in S_{l} \\
q \neq k}} H_{q}^{(l)} X_{q}^{(l)} \rho_{k q}^{(l)} \sum_{\substack{u=1 \\
(\neq),(l)}}^{K} \sum_{v \in S_{u}} \rho_{q v}^{(l),(u)} Z_{v}^{(u)}\right) \\
\quad\left[\left(1-w_{k,(3)}^{(i)}\left(1-w_{q,(2)}^{(l)} \sum_{\substack{u=1 \\
u \neq l}}^{K} \sum_{v \in S_{u}} \rho_{q v}^{(l),(u)} \rho_{v q}^{(u),(l)}\right)\right)\right. \\
-w_{k,(3)}^{(i)} \sum_{\substack{u=1 \\
u \neq i, l}}^{K} \sum_{v \in S_{u}} \rho_{k v}^{(l),(u)}\left(\rho_{v q}^{(u),(l)}\left(1-w_{v,(2)}^{(u)}\right)\right. \\
\left.\left.-w_{v,(2)}^{(u)} \sum_{\substack{n=1 \\
n \neq u, l}}^{K} \sum_{s \in S_{n}} \rho_{v s}^{(u),(n)} \rho_{s q}^{(n),(l)}\right)\right]
\end{gathered}
$$

After coherent combining using $\overline{H_{k}^{(i)}}$, the final output is

$$
\overline{H_{k}^{(i)}} Y_{k,(3)}^{(i)}=\left|H_{k}^{(i)}\right|^{2} X_{k}^{(i)} F+I_{3}^{\prime}+N_{3}^{\prime},
$$

where $I_{3}^{\prime}=\overline{H_{k}^{(i)}} I_{3}$ and $N_{3}^{\prime}=\overline{H_{k}^{(i)}} N_{3}$. Again, assuming that $H_{k 1}^{(i)}$ and $H_{k 2}^{(i)}$ are uncorrelated, we can obtain the variance of $N_{3}^{\prime}, \sigma_{N_{3}^{\prime}}^{2}$, and the variance of $I_{3}^{\prime}, \sigma_{I_{3}^{\prime}}^{2}$, respectively, as

$$
\sigma_{N_{3}^{\prime}}^{2}=\left|H_{k}^{(i)}\right|^{2} \sigma_{N_{3}}^{2}
$$

where

$$
\sigma_{N_{3}}^{2}=2 \sigma^{2}\left[N\left(1+w_{k,(3)}^{(i)} \sum_{\substack{l=1 \\ l \neq i}}^{K} \sum_{q \in S_{l}} \rho_{k q}^{(i),(l)} w_{q,(2)}^{(l)} \rho_{q k}^{(l),(i)}\right)^{2}\right.
$$$$
+N\left(w_{k,(3)}^{(i)}\right)^{2} \sum_{\substack{u=1 \\ u \neq i}}^{K} \sum_{r \in S_{u}}^{K}\left|\left(-\rho_{k r}^{(j),(u)}+\sum_{\substack{l=1 \\ l \neq i, u}}^{K} \sum_{q \in S_{l}} \rho_{k q}^{(i),(l)} \rho_{q r}^{(l),(i)} w_{q,(2)}^{(l)}\right)\right|^{2}
$$$$
+\left(w_{k,(3)}^{(i)}\right)^{2} \sum_{\substack{u=1 \\ u \neq i}}^{K} \sum_{r \in S_{u}} \sum_{\substack{c=1 \\ c \neq i, u}}^{K} \sum_{\substack{c \\ c \neq S_{c}}}\left(-\rho_{k r}^{(i),(u)}+\sum_{\substack{l \neq 1 \\ l \neq i, u}}^{K} \sum_{q \in S_{l}} \rho_{k q}^{(i),(l)} \rho_{q r}^{(l),(i)} w_{q,(2)}^{(l)}\right)
$$

$$
\overline{\left(-\rho_{k v}^{(i),(c)}+\sum_{\substack{l=1 \\ l \neq i, c}}^{K} \sum_{q \in S_{l}} \rho_{k q}^{(i),(l)} \rho_{q v}^{(l),(i)} w_{q,(2)}^{(l)}\right) \eta_{r v}^{(u),(c)}}
$$$$
+N\left(w_{k,(3)}^{(i)}\right)^{2} \sum_{r \in S_{i}}\left|\sum_{\substack{l=1 \\ l \neq i}}^{K} \sum_{q \in S_{l}} \rho_{k q}^{(i),(l)} \rho_{q r}^{(l),(i)} w_{q,(2)}^{(l)}\right|^{2}
$$$$
+2 w_{k,(3)}^{(i)} \mathcal{R e}\left(\sum_{\substack{u=1 \\ u \neq i}}^{K} \sum_{r \in S_{u}} \sum_{v \in S_{i}}\left(-\rho_{k r}^{(i),(u)}+\sum_{\substack{l=1 \\ l \neq i, u}}^{K} \sum_{q \in S_{l}} \rho_{k q}^{(i),(l)} \rho_{q r}^{(l),(i)} w_{q,(2)}^{(l)}\right)\right.
$$

$$
\left.\cdot \eta_{r v}^{(u),(i)} \overline{\sum_{\substack{l=1 \\ l \neq i}}^{K} \sum_{q \in S_{l}} \rho_{k q}^{(i),(l)} \rho_{q r}^{(l),(i)} w_{q,(2)}^{(l)}}\right)
$$$$
+2 w_{k,(3)}^{(i)} \mathcal{R} e\left(\sum_{\substack{u=1 \\ u \neq i}}^{K} \sum_{r \in S u} \eta_{r k}^{(u),(i)}\left(1+w_{k,(3)}^{(i)} \sum_{\substack{l=1 \\ l \neq i}}^{K} \sum_{q \in S_{l}} \rho_{k q}^{(i),(l)} w_{q,(2)}^{(l)} \rho_{q k}^{(l),(i)}\right)\right.
$$

$$
\left.\left.\overline{\left.\left(-\rho_{k r}^{(i),(u)}+\sum_{\substack{l=1 \\ l \neq i, u}}^{K} \sum_{q \in S_{l}} \rho_{k q}^{(i),(l)} \rho_{q r}^{(l),(i)} w_{q,(2)}^{(l)}\right)\right)}\right)\right]
$$

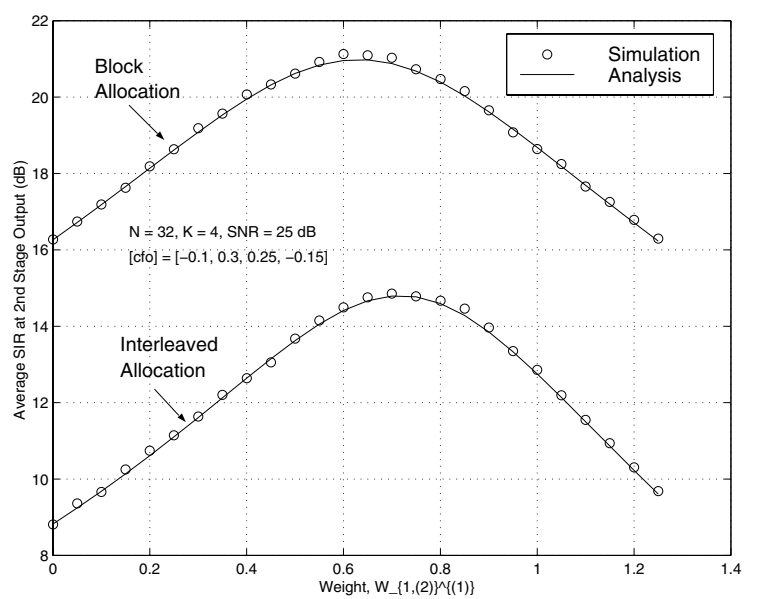

Fig. 3. Average SIR of the 1st user at the 2nd stage output of the proposed WLPIC scheme as a function of the weight on the 1st subcarrier, $w_{1,(2)}^{(1)}$. $K=4, N=32,16-\mathrm{QAM},\left[\epsilon_{1}, \epsilon_{2}, \epsilon_{3}, \epsilon_{4}\right]=[-0.1,0.3,0.25,-0.15]$. $\mathrm{SNR}=25 \mathrm{~dB}$, Interleaved and block allocation. Analysis vs simulation.

and

$$
\sigma_{I_{3}^{\prime}}^{2}=\left|H_{k}^{(i)}\right|^{2} \sigma_{I_{3}}^{2}
$$

where

$$
\begin{aligned}
\sigma_{I_{3}}^{2} & =2 \sum_{l=1}^{K} \sum_{\substack{q \in S_{l} \\
q \neq k}} \rho_{k q q}^{(i),(l)}\left[\left(1-w_{k,(3)}^{(i)}\left(1-w_{q,(2)}^{(l)} \sum_{\substack{u=1 \\
u \neq l}}^{K} \sum_{v \in S_{u}} \rho_{q v}^{(l),(u)} \rho_{v q}^{(u),(l)}\right)\right)\right. \\
& -w_{k,(3)}^{(i)} \sum_{\substack{u=1 \\
u \neq i, l}} \sum_{v \in S_{u}} \rho_{k v}^{(l),(u)}\left(\rho_{v q}^{(u),(l)}\left(1-w_{v,(2)}^{(u)}\right)\right. \\
& \left.\left.-w_{v,(2)}^{(u)} \sum_{\substack{n=1 \\
n \neq u, l}}^{K} \sum_{s \in S_{n}} \rho_{v s}^{(u),(n)} \rho_{s q}^{(n),(l)}\right)\right]\left.\right|^{2}
\end{aligned}
$$

As before, the average SIR on the $k$ th subcarrier of the $i$ th user at the 3rd stage output, $S I R_{k,(3)}^{(i)}$, can be obtained as

$$
S I R_{k,(3)}^{(i)}=\frac{2 F^{2}}{\sigma_{I_{3}}^{2}+\sigma_{N_{3}}^{2}} .
$$

\section{SIR Results and Discussions}

In Fig. 3, we plot the average SIR at the output of the 2nd stage as a function of weights $w_{k,(2)}^{(i)}$ obtained through both analysis (Eqn. 21) as well as simulations. The system parameters considered are: $N=32, K=4,\left[\epsilon_{1}, \epsilon_{2}, \epsilon_{3}, \epsilon_{4}\right]=$ $[-0.1,0.3,0.25,-0.15]$, and $\mathrm{SNR}=25 \mathrm{~dB}$. The channel model used is a one sample spaced two-ray equal-gain Rayleigh fading model. Average SIR for both block allocation as well as interleaved allocation are plotted.

The following observations can be made from Fig. 3. First, for the considered channel model and system parameters, block allocation results in a higher output SIR than interleaved allocation. Second, the match between the analytical SIR and simulated SIR is quite good implying that the uncorrelated assumption on $H_{k}^{(i)}$, s is reasonable. Third, the maximum average output SIR occurs at an optimum weight (maximum SIR of about $15 \mathrm{~dB}$ at $w_{k,(2)}^{(i)} \approx 0.7$ for interleaved allocation and a maximum SIR of about $21 \mathrm{~dB}$ at $w_{k,(2)}^{(i)} \approx 0.6$ for block allocation). This implies that the average SIR expressions in (21) and (31) can be maximized to obtain optimum weights. 


\section{Optimum Weights in Closed-FORM}

A. $w_{k,(2)}^{(i), o p t}$ in Closed-form

An expression for the optimum weights $w_{k,(2)}^{(i), o p t}$ can be obtained by differentiating (21) w.r.t. $w_{k,(2)}^{(i)}$ and equating to zero. Accordingly, we obtain the expression for $w_{k,(2)}^{(i),(o p t)}$ as

$$
w_{k,(2)}^{(i), o p t}=-\frac{\left(\beta_{1}+2 \beta_{2} \beta_{3}\right)}{\left(\beta_{1} \beta_{2}+2 \beta_{4}\right)},
$$

where

$$
\begin{aligned}
& \beta_{1}=\alpha_{1}-\sigma^{2}\left(\alpha_{6}+\alpha_{7}\right), \quad \beta_{2}=\sum_{\substack{l=1 \\
l \neq i}}^{K} \sum_{q \in S_{l}} \rho_{k q}^{(i),(l)} \rho_{q k}^{(l),(i)} \\
& \beta_{3}=\alpha_{2}+\sigma^{2} N, \quad \beta_{4}=\alpha_{3}+\sigma^{2}\left(N \alpha_{4}+\alpha_{5}\right), \\
& \alpha_{1}=2 \sum_{u=1}^{K} \sum_{\substack{r \in S_{u} \\
r \neq k}}\left|\rho_{k r}^{(i),(u)}\right|^{2}+2 \mathcal{R} e\left[\sum_{u=1}^{K} \sum_{\substack{r \in S_{u} \\
r \neq k}} \sum_{\substack{l=1 \\
l \neq i, u}}^{K} \sum_{q \in S_{l}} \rho_{k q}^{(i),(l)} \rho_{q r}^{(l),(u)}\right], \\
& \alpha_{3}=\sum_{u=1}^{K} \sum_{\substack{r \in S_{u} \\
r \neq k}}\left|\left(\rho_{k r}^{(i),(u)}+\sum_{\substack{l=1 \\
l \neq i, u}}^{K} \sum_{q \in S_{l}} \rho_{k q}^{(i),(l)} \rho_{q r}^{(l),(u)}\right)\right|^{2}, \\
& \alpha_{2}=\sum_{u=1}^{K} \sum_{\substack{r \in S_{u} \\
r \neq k}} \mid\left(\left.\rho_{k r}^{(i),(u)}\right|^{2}, \quad \alpha_{4}=\sum_{\substack{l=1 \\
l \neq i}}^{K} \sum_{q \in S_{l}}\left|\rho_{k q}^{(i),(l)}\right|^{2} .\right. \\
& \alpha_{5}=\sum_{\substack{l=1 \\
l \neq i}}^{K} \sum_{q \in S_{l}} \sum_{\substack{c=1 \\
c \neq i, l}}^{K} \sum_{v \in S_{c}} \rho_{k q}^{(i),(l)} \overline{\rho_{k v}^{(i),(c)}} \eta_{q v}^{(l),(c)}, \\
& \alpha_{6}=\mathcal{R} e\left[\sum_{\substack{l=1 \\
l \neq i}}^{K} \sum_{q \in S_{l}} \rho_{k q}^{(i),(l)} \eta_{q k}^{(l),(i)}\right] .
\end{aligned}
$$

B. $w_{k,(3)}^{(i), o p t}$ in Closed-form

Similarly, by differentiating (31) w.r.t. $w_{k,(3)}^{(i)}$ and equating to zero, we obtain the expression for the optimum weights $w_{k,(3)}^{(i), o p t}$, in closed-form, as

$$
w_{k,(3)}^{(i), o p t}=-\frac{\left(\gamma_{1}+2 \gamma_{2} \gamma_{3}\right)}{\left(\gamma_{1} \gamma_{2}+2 \gamma_{4}\right)},
$$

where

$$
\begin{aligned}
& \gamma_{1}=2 \psi_{2 b}+\sigma^{2}\left(2 N \psi_{4 a}+2 \psi_{4 f}+2 \psi_{4 g}\right), \quad \gamma_{3}=\psi_{2 c}+N \sigma^{2}, \\
& \gamma_{2}=\sum_{\substack{l=1 \\
l \neq i}}^{K} \sum_{q \in S_{l}} \rho_{k q}^{(i),(l)}\left[\rho_{q k}^{(l),(i)}\left(1-w_{q,(2)}^{(l)}\right)-w_{q,(2)}^{(l)} \sum_{\substack{n=1 \\
n \neq l, i}}^{K} \sum_{s \in S_{n}} \rho_{q s}^{(l),(n)} \rho_{s k}^{(n),(l)}\right] \\
& \gamma_{4}=\psi_{2 a}+\sigma^{2}\left(N \psi_{4 a}^{2}+N \psi_{4 b}+\psi_{4 c}+N \psi_{4 d}+2 \psi_{4 a} \psi_{4 f}\right), \\
& \psi_{2 c}=\sum_{u=1}^{K} \sum_{\substack{r \in S u \\
r \neq k}}\left|\rho_{k r}^{(i),(u)}\right|^{2}, \quad \psi_{4 a}=\sum_{\substack{l=1 \\
l \neq i}}^{K} \sum_{q \in S_{l}} \rho_{k q}^{(i),(l)} w_{q,(2)}^{(l)} \rho_{q k}^{(l),(i)}, \\
& \psi_{2 a}=\sum_{u=1}^{K} \sum_{\substack{r \in S u \\
r \neq k}} \mid \rho_{k r}^{(i),(u)}\left(1-w_{r,(2)}^{(u)} \sum_{\substack{n=1 \\
n \neq u}}^{K} \sum_{s \in S_{n}} \rho_{r s}^{(u),(n)} \rho_{s r}^{(n),(u)}\right) \\
& +\left.\sum_{\substack{l=1 \\
l \neq i, u}}^{K} \sum_{q \in S_{l}} \rho_{k l}^{(i),(u)}\left(\rho_{q r}^{(l),(u)}\left(1-w_{q,(2)}^{(l)}\right) w_{q,(2)}^{(l)} \sum_{\substack{n=1 \\
n \neq l, u}}^{K} \sum_{s \in S_{n}} \rho_{q s}^{(l),(n)} \rho_{s r}^{(n),(u)}\right)\right|^{2} \\
& \psi_{2 b}=\sum_{u=1}^{K} \sum_{\substack{r \in S u \\
r \neq k}}\left(-\left|\rho_{k r}^{(j),(u)}\right|^{2}\left(1-w_{r,(2)}^{(u)} \sum_{\substack{n=1 \\
n \neq u}}^{K} \sum_{s \in S_{n}} \rho_{r s}^{(u),(n)} \rho_{s r}^{(n),(u)}\right)\right. \\
& -\mathcal{R} e \overline{\rho_{k r}^{(i),(u)}} \sum_{\substack{l=1 \\
l \neq i, u}}^{K} \sum_{q \in S_{l}} \rho_{k q}^{(l),(u)}\left(\rho_{q r}^{(l),(u)}\left(1-w_{q,(2)}^{(l)}\right)\right. \\
& \left.\left.\left.-w_{q,(2)}^{(l)} \sum_{\substack{n=1 \\
n \neq l, u}}^{K} \sum_{s \in S_{n}} \rho_{q s}^{(l),(n)} \rho_{s r}^{(n),(u)}\right)\right]\right) \text {, }
\end{aligned}
$$

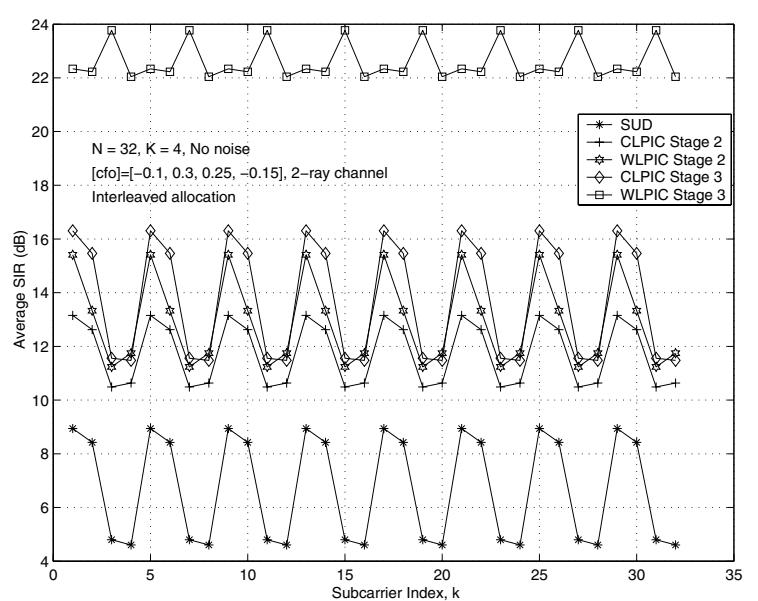

Fig. 4. Average SIR as a function of subcarrier index, $k$, for different detectors. $N=32, K=4,\left[\epsilon_{1}, \epsilon_{2}, \epsilon_{3}, \epsilon_{4}\right]=[-0.1,0.3,0.25,-0.15]$. No noise $\left(\sigma^{2}=0\right)$. Interleaved allocation. Analysis.

$$
\begin{aligned}
& \psi_{2 c}=\sum_{u=1}^{K} \sum_{\substack{r \in S u \\
r \neq k}}\left|\rho_{k r}^{(i),(u)}\right|^{2}, \quad \psi_{4 a}=\sum_{\substack{l=1 \\
l \neq i}}^{K} \sum_{q \in S_{l}} \rho_{k q}^{(i),(l)} w_{q,(2)}^{(l)} \rho_{q k}^{(l),(i)}, \\
& \psi_{4 c}=\sum_{\substack{u=1 \\
u \neq i}}^{K} \sum_{r \in S_{u}} \sum_{\substack{c=1 \\
c \neq i, u}}^{K} \sum_{v \in S_{c}}\left(-\rho_{k r}^{(i),(u)}+\sum_{\substack{l=1 \\
l \neq i, u}}^{K} \sum_{q \in S_{l}} \rho_{k q}^{(i),(l)} \rho_{q r}^{(l),(i)} w_{q,(2)}^{(l)}\right) \\
& \overline{\left(-\rho_{k v}^{(i),(c)}+\sum_{\substack{l=1 \\
l \neq i, c}}^{K} \sum_{q \in S_{l}} \rho_{k q}^{(i),(l)} \rho_{q v}^{(l),(i)} w_{q,(2)}^{(l)}\right)} \eta_{r v}^{(m)},(c), \\
& \psi_{4 d}=\sum_{r \in S_{i}}\left|\sum_{\substack{l=1 \\
l \neq i}}^{K} \sum_{q \in S_{l}} \rho_{k q}^{(i),(l)} \rho_{q r}^{(l),(i)} w_{q,(2)}^{(l)}\right|^{2}, \\
& \psi_{4 f}=\mathcal{R} e\left[\sum_{\substack{u=1 \\
u \neq i}}^{K} \sum_{r \in S_{u}} \eta_{r v}^{(u),(i)} \overline{\left(-\rho_{k r}^{(i),(u)}+\sum_{\substack{l=1 \\
l \neq i, u}}^{K} \sum_{q \in S_{l}} \rho_{k q}^{(i),(l)} \rho_{q r}^{(l),(i)} w_{q,(2)}^{(l)}\right)}\right] \\
& \psi_{4 g}=\mathcal{R} e\left[\sum_{\substack{u=1 \\
u \neq i}}^{K} \sum_{r \in S_{u}} \sum_{v \in S_{i}}\left(-\rho_{k r}^{(i),(u)}+\sum_{\substack{l=1 \\
l \neq i, u}}^{K} \sum_{q \in S_{l}} \rho_{k q}^{(i),(l)} \rho_{q r}^{(l),(i)} w_{q,(2)}^{(l)}\right)\right. \\
& \eta_{r v}^{(u),(i)} \overline{\left.\sum_{\substack{l=1 \\
l \neq i}}^{K} \sum_{q \in S_{l}} \rho_{k q}^{(i),(l)} \rho_{q r}^{(l),(i)} w_{q,(2)}^{(l)}\right]}
\end{aligned}
$$

\section{RESUlTS AND Discussion}

In this section, we present the numerical and simulation results of the average SIR and BER performance of the proposed WLPIC scheme. The channel model used throughout this section is a one sample spaced two-ray equal-gain Rayleigh fading model. In Figs. 4 and ??, we plot the analytically computed average SIR as a function of the subcarrier index $k=1,2, \cdots, N$ under no noise condition (i.e., $\sigma^{2}=0$ ) for $a$ ) SUD, b) 2nd and 3rd stages of the CLPIC scheme (i.e., $w_{k,(2)}^{(i)}=w_{k,(3)}^{(i)}=1$ ), and c) 2nd and 3rd stages of the WLPIC scheme, for an uplink OFDMA system with $N=32$ subcarriers, $K=4$ users, and CFOs of the different users $\left[\epsilon_{1}, \epsilon_{2}, \epsilon_{3}, \epsilon_{4}\right]=[-0.1,0.3,0.25,-0.15]$.

From Fig. 4 it can be seen that the SUD gives the least SIRs in all subcarriers since no interference cancellation is performed. When interference cancellation is performed using CLPIC scheme (where unity weights are used), the 2nd stage output SIR improves significantly compared to that of SUD. The CLPIC 3rd stage output SIR improves further compared to the CLPIC 2nd stage output SIR. The WLPIC scheme (where the optimized weights derived in Sec. V are used) performs 


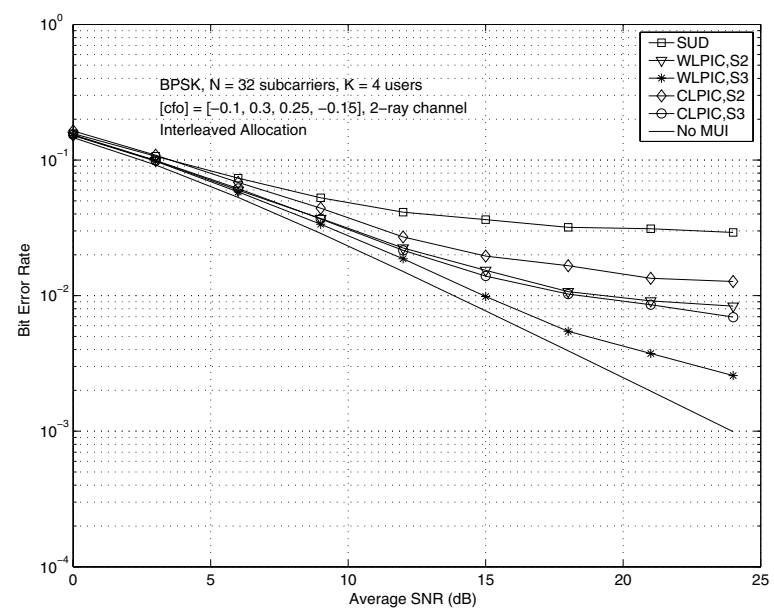

Fig. 5. Bit error rate performance of the proposed WLPIC scheme for BPSK. $N=32, K=4,\left[\epsilon_{1}, \epsilon_{2}, \epsilon_{3}, \epsilon_{4}\right]=[-0.1,0.3,0.25,-0.15]$. Interleaved allocation. Simulation.

significantly better than both SUD as well as CLPIC. For example, the 3rd stage of the WLPIC results in an average SIR of about $23 \mathrm{~dB}$ on all the subcarriers which is significantly larger than those of the other detectors. Thus the performance benefit of using the optimized weights in WLPIC instead of unity weights in CLPIC or zero weights in SUD is clearly evident in Fig. 4.

For the same set of parameters in Fig. 4, we plot the simulated BER performance of SUD, CLPIC (2nd and 3rd stages) and WLPIC (2nd and 3rd stages) in Fig. 5 for BPSK. The single user performance (no MUI) is also shown for comparison purposes. From Fig. 5, it can be seen that the proposed WLPIC scheme results in significantly better BER performance than both the SUD as well as the CLPIC scheme. The 3rd stage of the WLPIC scheme is found to approach the single user (no MUI) performance. Similar BER performance results for the case of 16-QAM are shown in Fig. 6 for $N=64$ subcarriers and $K=4$ users. The CFO values used in Fig. 6 are $\left[\epsilon_{1}, \epsilon_{2}, \epsilon_{3}, \epsilon_{4}\right]=[0.1,-0.1,-0.05,0.05]$. From Fig. 6, it can be observed that even the 2nd stage of the WLPIC scheme performs close to the single user (no MUI) performance. This is because the CFO values considered in Fig. 6 and hence the MUI caused are small compared to those considered in Fig. 5. The 3rd stage of the WLPIC in this case is found to be almost same as the single user (no MUI) performance illustrating the effectiveness of the proposed cancellation approach. We have observed similar SIR and BER performance improvement for the case of block allocation as well.

\section{CONCLUSiONS}

We presented the design and analysis of an interference cancellation scheme for MUI mitigation in OFDMA uplinks. The proposed scheme is a multistage linear parallel interference canceller which uses the soft values of the DFT output in the receiver to generate an estimate of the MUI for cancellation. We scaled the MUI estimate by weights before cancellation. We derived expressions for the output SIR at the 2nd and 3rd stages of the proposed scheme. While these SIR expressions quantified the improvement in output SIR from one stage to the next, they were also used to obtain the optimum weights,

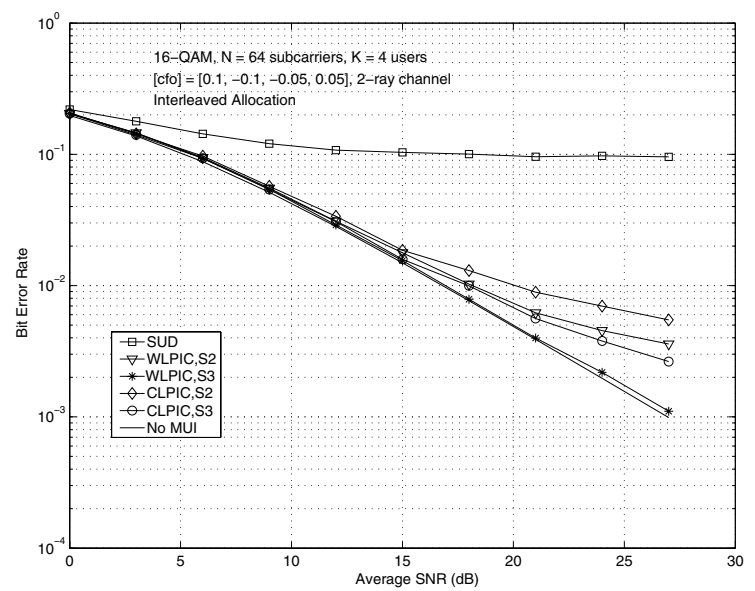

Fig. 6. Bit error rate performance of the proposed WLPIC scheme for 16-QAM. $N=64, K=4,\left[\epsilon_{1}, \epsilon_{2}, \epsilon_{3}, \epsilon_{4}\right]=[0.1,-0.1,-0.05,0.05]$. Interleaved allocation. Simulation.

in-closed form. The proposed scheme was shown to effectively cancel the MUI caused by the other user CFOs. As further extension to this work, we are investigating the following: a) a detailed performance/complexity comparison of the proposed scheme with the circular convolution based scheme in [9], b) coded BER performance, and c) performance in the presence of imperfect $\mathrm{CFO}$ estimates at the receiver.

\section{REFERENCES}

[1] K. Kim, Y. Han, and S-L. Kim, "Joint subcarrier and power allocation in uplink OFDMA systems," IEEE Comm. Letters, vol. 9, no. 6, pp. 526-528, June 2005

[2] Z. R. Cao, U. Tureli, and Y-D. Yao, "Deterministic multiuser carrier frequency offset estimation for interleaved OFDMA uplink," IEEE Trans. on Commun., vol. 52, no. 9, pp. 1585-1594, 2004.

[3] H. Wang and B. Chen, "Asymptotic distributions and peak power analysis for uplink OFDMA," Proc. IEEE ICASSP'2004, May 2004.

[4] M. O. Pun, C.-C. J. Juo, and M. Morelli, "Joint synchronization and channel estimation in uplink OFDMA systems," Proc. IEEE ICASSP'2005, March 2005 .

[5] A. M. Tonello, N. Laurenti, and S. Pupolin, "Analysis of the uplink of an asynchronous multiuser DMT OFDMA system impaired by time offsets, frequency offsets, and multipath fading," Proc. IEEE VTC'2000 (Fall), vol. 3, pp. 1094-1099, October 2000.

[6] J. Choi, C. Lee, H. W. Jung, and Y. H. Lee, "Carrier frequency offset compensation for uplink of OFDM-FDMA systems," IEEE Commun. Letters, vol. 4, no. 12, pp. 414-416, December 2000.

[7] Z. Cao, U. Tureh, and Y. D. Yao, "Analysis of two receiver schemes for interleaved OFDMA uplink signals," 36th Asilomar Conf. on Signals, Systems and Computers, vol. 2, pp.1818-1821, November 2002.

[8] R. Fantacci, D. Marabissi, and S. Papini, "Multiuser interference cancellation receivers for OFDMA uplink communications with carrier frequency offset," Proc. IEEE GLOBECOM'04, pp. 2808-2812, 2004.

[9] D. Huang and K. B. Letaief, "An interference cancellation scheme for carrier frequency offsets correction in OFDMA systems," IEEE Trans. on Commun., vol. 53, no. 7, pp. 1155-1165, July 2005.

[10] D. Sreedhar and A. Chockalingam, "MMSE receiver for multiuser interference cancellation in uplink OFDMA," to appear in Proc. IEEE VTC'06 Spring, 2006.

[11] T. Pollet, M. V. Bladel, and M. Moeneclaey, "BER sensitivity of OFDM systems to carrier frequency offset and Weiner phase noise," IEEE Trans. Commun., vol. 43, pp. 191-193, February/March/April 1995.

[12] L. Rugini and P. Banelli, and S. Cacopardi, "Probability of error of OFDM systems with carrier offset in frequency-selective fading channels," Proc. IEEE GLOBECOM'2004, pp. 3289-3293, 2004. 